\title{
HEMANGIOMA HEPÁTICO GIGANTE
}

\section{GIANT HEPATIC HEMANGIOMA}

\author{
Ramírez Sotomayor, Julio*; Adorno, Carlos ${ }^{* *}$; \\ Benza Bareiro, Alejandra ${ }^{* * *}$; González, Mercedes ${ }^{* * *}$
}

\section{RESUMEN}

Los hemangiomas hepáticos son los tumores benignos más frecuentes, comúnmente se presentan en mujeres y son considerados gigantes cuando su diámetro supera $4 \mathrm{~cm}^{1}$.

Se manifiestan con dolor abdominal y efecto de masa. Estos tumores pueden ser manejados por observación, enucleación, resección, y embolización ${ }^{2}$.

Palabras clave: hemangiomas, tumores benignos, efecto de masa.

\section{ABSTRACT}

Liver haemangiomas are the most common benign tumours, commonly presented in women and considered giant when their diameter surpasses $4 \mathrm{~cm}^{1}$.

They manifest with abdominal pain and mass effect. These tumours can be managed by observation, enucleation, resection, and embolisation ${ }^{2}$.

Key words: haemangiomas, bening tumors, efecto de masa.

\section{INTRODUCCIÓN}

La gran mayoría de los hemangiomas son de pequeño tamaño y asintomáticos por lo cual suelen no tener mayor relevancia clínica. Sin embargo, existen lesiones voluminosas, ocasionalmente múltiples, que generan sintomatología propia ${ }^{3}$.

Por lo general son asintomáticos, pero los hemangiomas gigantes pueden producir molestias abdominales, sangrado o síntomas obstructivos ${ }^{1}$.

Si los hemangiomas son pequeños y asintomáticos no requieren seguimiento luego del diagnóstico. Los pacientes con hemangiomas gigantes y sintomáticos deben ser referidos a centros de mayor complejidad para un potencial tratamiento quirúrgico ${ }^{4-5}$.

\section{CASO CLÍNICO}

Paciente de sexo femenino de 49 años de edad, Cuadro de 4 días de evolución de dolor en hipocondrio derecho de inicio insidioso, tipo puntada, de moderada intensidad que cede parcialmente con la ingesta de analgésicos comunes. Refiere además sensación febril acompañado de escalofríos y náuseas sin vómitos.

ABDOMEN: poco depresible, se palpa tumoración a nivel de epigastrio de consistencia sólida-elástica de aproximadamente $6 \mathrm{~cm}$ de diámetro, doloroso a la palpación profunda.

ECOGRAFÍA ABDOMINAL: HÍGADO: de tamaño aumentado, parénquima heterogéneo a expensas de imagen mixta, bien delimitada, de borde regular, visualizada en lóbulo izquierdo, mide 183 × 80 x $170 \mathrm{~mm}$, sin vascularización al Doppler color, con ecogenicidad aumentada.

TAC: se observa imagen heterogénea que ocupa todo el lóbulo izquierdo del hígado.

Se indica laparotomía exploradora con el diagnóstico probable de hemangioma hepático.

Se realiza laparotomía exploradora donde se confirma el diagnóstico de hemangioma hepático.

Hallazgos: a la apertura de cavidad se constata hemangioma gigante de $16 \times 12 \mathrm{~cm}$ con múltiples focos de necrosis.

Debido al gran tamaño del tumor se realiza lobectomía izquierda por laparotomía.

Se envía pieza a anatomía patológica donde se informa tejido hepático con abundantes luces vasculares dilatadas no anastomosadas, separadas por tejido fibroso, algunas ocupadas con trombos hemáticos. Focos de hialinización y calcificación.

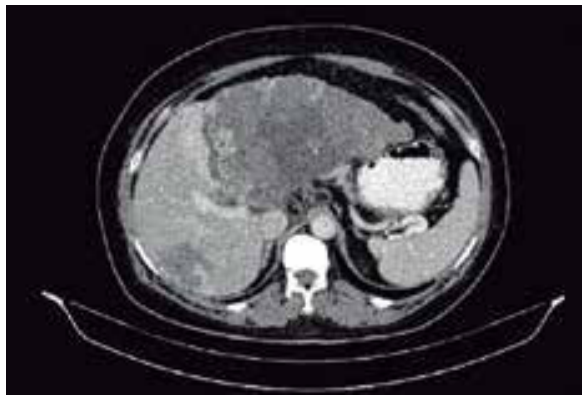

FIG. 1. TAC con contraste de abdomen y pelvis (foto archivo de la autora).

* Jefe de Servicio.

** Jefe de Sala.

${ }^{* * *}$ Residente de segundo año.

Servicio de Cirugía General. Hospital Nacional de Itauguá.

Autora correspondiente: Dra. Ma. Alejandra Benza Bareiro - Dirección: 18 de Julio casi Pizarro 2223 (Asunción-Paraguay)- Tel: +595981936523

- E-mail: alebenza@gmail.com

Articulo recibido: $10 / 11 / 2016$

Articulo aceptado: 21/11/2016 


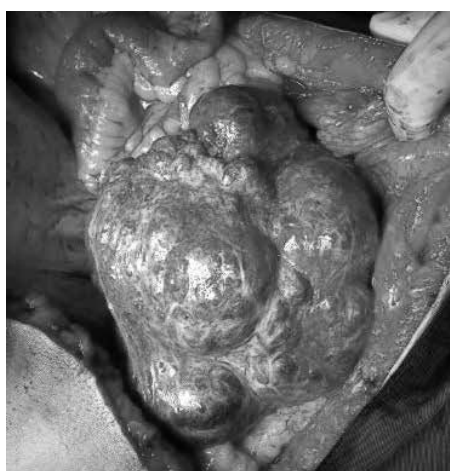

FIG. 2. Pieza operatoria (foto archivo de la autora).

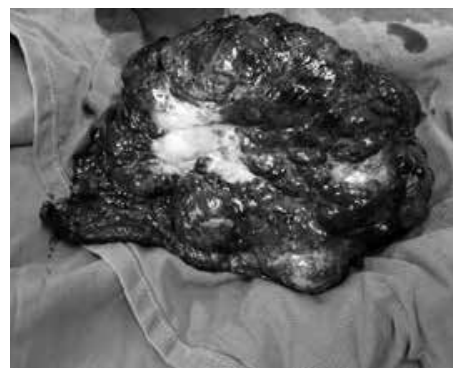

FIG. 3. Pieza operatoria (foto archivo de la autora).

\section{DISCUSIÓN}

La mayoría de los pacientes tienen un excelente pronóstico debido a la naturaleza benigna del tumor ${ }^{6-7}$.

El tratamiento del hemangioma hepático depende fundamentalmente de su tamaño, el número de lesiones, la presencia o no de síntomas y la edad de aparición ${ }^{8}$.

Las opciones de tratamiento del hemangioma hepático, incluyendo el hemangioma hepático gigante, son la observación, enucleación, resección hepática y embolización arterial transcatéter; recientemente la ablación por radiofrecuencia progresiva ha sido utilizada para el tratamiento de los hem-

\section{REFERENCIAS}

1. Oak CY, Jun CH, Cho EA, Lee DH, Cho SB, Park CH, Joo YE Kim HS, Rew JS, Choi SK. Hepatic Hemangioma with KasabachMerritt Syndrome in an Adult Patient. Korean J Gastroenterol. 2016 Apr 25;67(4):220-223. doi: 10.4166/kig.2016.67.4.220.

2. Ortiz-Bayliss AB, Martínez-Mier G, Alvarado-Arenas RA, Lajud-Barquín FA. Surgical treatment of liver haemangiomas in a third level hospital in south-east Mexico. Cir Cir. 2016 Apr 27. pii: S0009-7411(16)30021-4. doi: 10.1016/j.circir.2016.03.009.

3. Benavides C, Garcia C, Rubilar P, Covacevich S, Perales C, Ricarte F, Stock R. Hemangiomas hepáticos. Rev. Chilena de Cirugía. Vol 58 - No 3, Junio 2006; págs. 194-198.

4. Strauss E, Ferreira Ade S, França AV, Lyra AC, Barros FM, Silva I, Garcia JH, Parise ER. Diagnosis and treatment of benign liver nodules: Brazilian Society of Hepatology (SBH) recommendations. Arq Gastroenterol. 2015 Dec;52 Suppl 1:47-54. doi: 10.1590/S0004-28032015000500003.

5. Choi J, Lee YJ, Hwang DW, Chon SH, Nagpal A, Park KM. Surgical treatment of giant hepatic hemangiomas: technical point of view. Am Surg. 2011 Jan;77(1):48-54.

6. Zhao W, Guo X, Dong J. Spontaneous rupture of hepatic hemangioma: a case report and literature review. Int J Clin Exp Pathol. 2015 Oct 1;8(10):13426-8. eCollection 2015.

7. Taseva A, Tasev V, Bonev S, Dimitrova V. Liver hemangiomas-surgical point of view. Khirurgiia (Sofiia). 2014;(2):63-8.

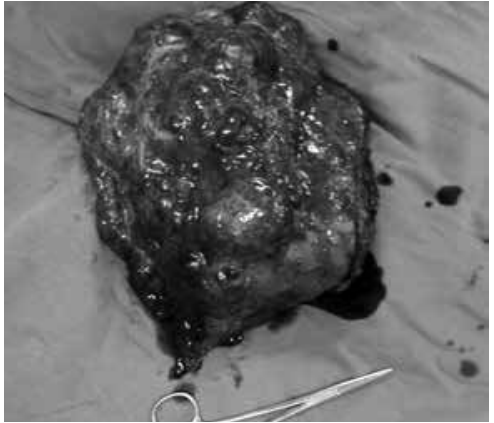

FIG. 4. Pieza operatoria (foto archivo de la autora).

angiomas hepáticos gigantes, mostrando buenos resultados 9 .

La cirugía se reserva para casos excepcionales en que existe un crecimiento sostenido y no se puede descartar la naturaleza de la lesión, existen síntomas secundarios a su crecimiento, o se desarrolla un síndrome de KasabachMerritt (trombocitopenia, alteraciones de la coagulación y hemangioma gigante) ${ }^{10}$.

La enucleación es el tratamiento quirúrgico más frecuente y es suficiente para el control de estas lesiones, sin embargo, se asocia a sangrado intraoperatorio importante. Una hepatectomía reglada, con control vascular previo a la transección parenquimatosa puede ser más segura ${ }^{11}$.

Los factores más importantes asociados con las complicaciones quirúrgicas son el tamaño del tumor y la presencia de síntomas ${ }^{12}$.

Se interviene quirúrgicamente el $2 \%$ de los hemangiomas hepáticos diagnosticados, las indicaciones más frecuentes para la cirugía son el desarrollo de complicaciones como la ruptura y el sangrado intraperitoneal que tienen una mortalidad de $20 \%$ en los hemangiomas grandes ${ }^{13}$.

La morbimortalidad de la resección de estos tumores es muy baja, ya que se realiza en hígado sano, es limitada, se realiza en personas jóvenes y sin enfermedades concomitantes ${ }^{14}$.

8. H. Zou, J. Yan, Y.X. Wu, et al. The new technology of enhanced radiofrequency ablation is safe and effective for treating giant hepatic hemangioma. Zhonghua Gan Zang Bing Za Zhi, 20 (2012), pp. 261-265

9. Hugh T, Poston G. Benign Liver tumors and masses. En: Surgery of the liver and biliary tract Third Edition, vol II. Blumgart L, Fong Y (eds). London: Editorial WB Saunders Company Ltd, 2002: 1397-1422.

10. Yamamoto T, Kawarada Y. La ruptura espontánea del hemangioma del hígado: el tratamiento con el transcateter para embolización arterial hepática. Es J Gastroenterology. 2011;86:1645.

11. Yoon S, Charny C, Fong Y, Jarnagin W, Schwartz L, Blumgart L, et al. Diagnosis, management, and outcomes of 115 patients with hepatic hemangioma. J Am Coll Surg 2003; 197: 392-402.

12. H.Y. Ho, T.H. Wu, M.C. Yu, et al. Surgical management of giant hepatic hemangiomas: Complications and review of the literature. Chang Gung Med J, 35 (2012), pp. 70-78

13. Kadry Z, Mentha G, Cereda JM. La ruptura del hemangioma como una manifestación del hemangioma cavernoso hepático gigante. J Hepatol. 2000;32:358.

14. Ramia JM, Muffak K, Villar J, Garrote D, Ferrón JA. Tumores hepáticos sólidos benignos. Cirugía Española. Volume 77, Issue 5, May 2005, Pages 247-253 